Preprints of the

Max Planck Institute for

Research on Collective Goods

Bonn 2008/45

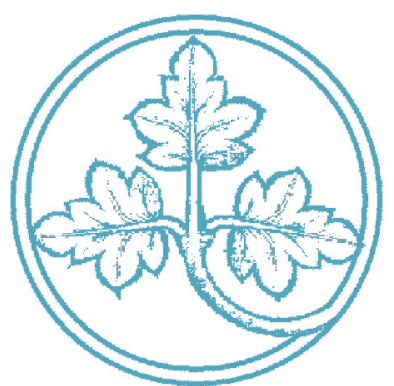

A Note on Deaton's

Theorem on the

Undesirability of Nonuniform Excise Taxation

Martin Hellwig

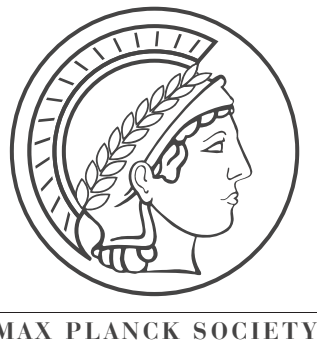




\section{A Note on Deaton's Theorem on the Undesirability of Nonuniform Excise Taxation}

Martin Hellwig

December 2008 


\title{
A Note on Deaton's Theorem on the Undesirability of Nonuniform Excise Taxation*
}

\author{
Martin F. Hellwig \\ Max Planck Institute for Research on Collective Goods \\ Kurt-Schumacher-Str. 10, D-53113 Bonn, Germany \\ hellwig@coll.mpg.de
}

December 2, 2008

\begin{abstract}
The paper provides an extension and a new proof of Deaton's theorem on the undesirability of nonuniform excise taxation when income taxes are affine and preferences over consumption goods are separable from labour-leisure choices, homothetic, and identical across agents.

Key Words: Optimal Excise Taxes, Atkinson-Stiglitz Theorem, Deaton's Theorem

JEL Classification: $\mathrm{H} 21$
\end{abstract}

\section{Introduction}

A well known result of Atkinson and Stiglitz (1976) shows that, under certain homogeneity and separability assumptions on preferences, an optimal system of taxes for public-sector funding or for redistribution relies on direct taxation only. Deaton (1979) has shown that, if income tax schedules are restricted to be affine, the conclusion of Atkinson and Stiglitz remains valid under the additional assumption that preferences are homothetic in the consumption goods. Deaton's result plays a role in the literature on the undesirability of capital income taxation in the presence of an affine income tax schedule, e.g., Werning (2007).

This note provides a new proof and an extension of Deaton's result. Whereas Deaton focussed on optimal affine income taxes, the analysis here

${ }^{*}$ I thank Felix Bierbrauer, Christoph Engel, and Andreas Nicklisch for very helpful comments on a previous draft of this note. 
shows that,under his assumptions, any tax system with affine income taxes and nonuniform linear excise taxes is Pareto-dominated by a tax system with affine income taxes only and no excise taxes at all. The proof uses an argument that Kaplow (2006) and Laroque (2005) have recently developed to simplify the proof and extend the scope of the Atkinson-Stiglitz theorem itself. For Deaton's theorem, the argument provides a more direct access to the relation between the assumption that preferences are homothetic in the consumption goods and the requirement that the income tax schedule be affine.

\section{The Setup}

Consider an economy with $m$ final goods, one intermediate good, and labour. The final goods are produced from the intermediate good, the intermediate good from labour. Different agents have different productivities. One unit of final good $i$ requires $p_{i}$ units of the intermediate good. One unit of the intermediate good requires $\frac{1}{t}$ units of labour from an agent with productivity $t$. An agent with productivity $t$ who has final-good consumption $c_{1}, \ldots, c_{m}$ and who provides the labour input $\ell=\frac{y}{t}$ that is required to produce the amount $y$ of the intermediate good, gets the utility $u\left(c_{1}, \ldots, c_{m}, \frac{y}{t}\right)$. The utility function $u$ is continuously differentiable, increasing in $c_{1}, \ldots, c_{m}$, decreasing in $\ell=\frac{y}{t}$, and strictly quasi-concave.

An allocation is a mapping $t \rightarrow\left(c_{1}(t), \ldots, c_{m}(t), y(t)\right)$ that indicates for each $t$ how much of each final good an agent with productivity parameter $t$ gets to consume and how much of the intermediate good he has to supply. The allocation $\left(c_{1}(\cdot), \ldots, c_{m}(\cdot), y(\cdot)\right)$ involves the aggregate consumption $\int c_{i}(t) d F(t)$ of final good $i$ and aggregate production $\int y(t) d F(t)$ of the intermediate good, where $F$ is the cross-section distribution of $t$ in the population. The allocation is feasible if

$$
\sum_{i=1}^{m} p_{i} \int c_{i}(t) d F(t) \leq \int y(t) d F(t) .
$$

If the inequality in (1) is strict, I will say that the allocation is strictly feasible.

An allocation $\left(c_{1}(\cdot), \ldots, c_{m}(\cdot), y(\cdot)\right)$ is implementable by the income tax schedule $T(\cdot)$ with consumer prices $q_{1}, \ldots, q_{m}$ if, for every $t,\left(c_{1}(t), \ldots, c_{m}(t), y(t)\right)$ maximizes the utility of a person with productivity parameter $t$ under the 
budget constraint

$$
\sum_{i=1}^{m} q_{i} c_{i} \leq y-T(y) .
$$

Trivially, an allocation that is implementable by an income tax schedule $T(\cdot)$ with consumer prices $q_{1}, \ldots, q_{m}$ is also incentive-compatible in the sense that

$$
u\left(c_{1}(t), \ldots, c_{m}(t), \frac{y(t)}{t}\right) \geq u\left(c_{1}\left(t^{\prime}\right), \ldots, c_{m}\left(t^{\prime}\right), \frac{y\left(t^{\prime}\right)}{t}\right)
$$

for all $t$ and $t^{\prime}$.

Atkinson and Stiglitz (1976), as well as Kaplow (2006) and Laroque (2005), assume that $u$ takes the separable form

$$
u\left(c_{1}, \ldots, c_{m}, \frac{y}{t}\right)=v\left(\varphi\left(c_{1}, \ldots, c_{m}\right), \frac{y}{t}\right),
$$

where $v$ is continuous and $\varphi$ is continuous and increasing. Under this assumption, the incentive compatiblity condition (3) is equivalent to the requirement that

$$
v\left(w(t), \frac{y(t)}{t}\right) \geq v\left(w\left(t^{\prime}\right), \frac{y\left(t^{\prime}\right)}{t}\right),
$$

where, for each $t$,

$$
w(t):=\varphi\left(c_{1}(t), \ldots, c_{m}(t)\right) .
$$

Incentive compatibility depends only on the output requirement $y(t)$ and the subutility $w(t)$ that is given by (6). Which consumption vector is used to achieve the subutility $w(t)$ is irrelevant for incentive compatibility, but matters for feasibility. If final-goods consumption vectors can be rearranged so as to reduce resource costs while achieving the same subutility levels, one can replace the incentive-compatible and feasible allocation $\left(c_{1}(\cdot), \ldots, c_{m}(\cdot), y(\cdot)\right)$ by a payoff-equivalent allocation that is also incentivecompatible and strictly feasible. If the surplus that is thereby achieved can be used to raise utility levels without upsetting incentive compatibility, the allocation $\left(c_{1}(\cdot), \ldots, c_{m}(\cdot), y(\cdot)\right)$ is strictly dominated. By the taxation principle of Hammond (1979) and Guesnerie (1995), the payoff-equivalent and the dominating allocation can both be implemented by an income tax schedule with the consumer prices $p_{1}, \ldots, p_{m}$.

\section{A Generalization of Deaton's Theorem}

In the preceding argument, there is no restriction on the tax schedule that implements the dominating allocation. To deal with the additional restriction that the tax schedule must be affine, Deaton imposes the additional 
assumption that $\varphi$ is homothetic. Under this assumption, he shows that any allocation that maximizes welfare subject to feasibility and implementability by an affine income tax schedule must involve consumer prices equal to the producer prices $p_{1}, \ldots, p_{m}$. The following result shows that, in fact, any allocation that is implemented by an affine income tax schedule with consumer prices that are not proportional to the producer prices is Pareto-dominated. This generalizes Deaton's theorem in the same way that Kaplow (2006) and Laroque (2005) generalized the Atkinson-Stiglitz theorem.

Theorem 1 Assume that $u$ takes the separable form (4) where $\varphi$ is homothetic. Let $\left(c_{1}(\cdot), \ldots, c_{m}(\cdot), y(\cdot)\right)$ be any feasible allocation that is implementable by an affine income tax schedule $T(\cdot)$ with consumer prices $q_{1}, \ldots, q_{m}$. If the consumer prices $q_{1}, \ldots, q_{m}$ are not proportional to the producer prices $p_{1}, \ldots, p_{m}$, the allocation $\left(c_{1}(\cdot), \ldots, c_{m}(\cdot), y(\cdot)\right)$ is strictly Paretodominated by another feasible allocation that can be implemented by an affine income tax schedule $\hat{T}(\cdot)$ with consumer prices equal to the producer prices $p_{1}, \ldots, p_{m}$.

Proof. I first show that,if the consumer prices $q_{1}, \ldots, q_{m}$ are not proportional to the producer prices $p_{1}, \ldots, p_{m}$, there exists a strictly feasible allocation which provides participants with the same payoffs as the allocation $\left(c_{1}(\cdot), \ldots, c_{m}(\cdot), y(\cdot)\right)$. For any $t$, let $\left(\hat{c}_{1}(t), \ldots, \hat{c}_{m}(t)\right)$ be the solution to the problem

$$
\min _{c_{1}, \ldots, c_{m}} \sum_{i=1}^{m} p_{i} c_{i} \quad \text { s.t. } \quad \varphi\left(c_{1}, \ldots, c_{m}\right)=w(t),
$$

where $w(t)$ is given by $(6)$. The allocation $\left(\hat{c}_{1}(\cdot), \ldots, \hat{c}_{m}(\cdot), y(\cdot)\right)$ is payoff equivalent to the allocation $\left(c_{1}(\cdot), \ldots, c_{m}(\cdot), y(\cdot)\right)$. Moreover, for any $t$, one has

$$
\sum_{i=1}^{m} p_{i} \hat{c}_{i}(t) \leq \sum_{i=1}^{m} p_{i} c_{i}(t) .
$$

Indeed, because $u$ is strictly quasi-concave and differentiable and because the consumer price ratios $q_{i} / q_{j}$ are not all the same as the producer price ratios $p_{i} / p_{j}$, the inequality in (8) must be strict. If the allocation $\left(c_{1}(\cdot), \ldots, c_{m}(\cdot), y(\cdot)\right)$ is feasible, the allocation $\left(\hat{c}_{1}(\cdot), \ldots, \quad \hat{c}_{m}(\cdot), y(\cdot)\right)$ must be strictly feasible.

I next show that the allocation $\left(\hat{c}_{1}(\cdot), \ldots, \hat{c}_{m}(\cdot), y(\cdot)\right)$ can be implemented by an affine income tax schedule $\hat{T}(\cdot)$, with consumer prices equal to $p_{1}, \ldots, p_{m}$. I begin by specifying the schedule $\hat{T}(\cdot)$. The assumption that $\left(c_{1}(\cdot), \ldots, c_{m}(\cdot), y(\cdot)\right)$ can be implemented by the tax schedule $T(\cdot)$ with consumer prices $q_{1}, \ldots, q_{m}$ implies that, for each $t$, the vector $\left(c_{1}(t), \ldots, c_{m}(t)\right)$ maximizes $\varphi\left(c_{1}, \ldots, c_{m}\right)$ 
under the constraint that (2) hold for $y=y(t)$. By the homotheticity of $\varphi$, it follows that $\left(c_{1}(t), \ldots, c_{m}(t)\right)$ and $w(t)$ take the form

$$
c_{i}(t)=(y(t)-T(y(t))) \gamma_{i}, \text { for } i=1, \ldots, m,
$$

and

$$
w(t)=\psi(y(t)-T(y(t))) \varphi\left(\gamma_{1}, \ldots, \gamma_{m}\right)
$$

where $\left(\gamma_{1}, \ldots, \gamma_{m}\right)$ maximizes $\varphi\left(c_{1}, \ldots, c_{m}\right)$ under the constraint $\sum_{i=1}^{m} q_{i} c_{i} \leq$ 1. Also by the homotheticity of $\varphi$, the definition of $\left(\hat{c}_{1}(t), \ldots, \hat{c}_{m}(t)\right)$ as the solution to problem $(7)$ implies that $\left(\hat{c}_{1}(t), \ldots, \hat{c}_{m}(t)\right)$ takes the form

$$
\hat{c}_{i}(t)=(y(t)-T(y(t))) \hat{\gamma}_{i}, \text { for } i=1, \ldots, m,
$$

where $\left(\hat{\gamma}_{1}, \ldots, \hat{\gamma}_{m}\right)$ minimizes $\sum_{i=1}^{m} p_{i} c_{i}$ under the constraint

$$
\varphi\left(c_{1}, \ldots, c_{m}\right)=\varphi\left(\gamma_{1}, \ldots, \gamma_{m}\right) .
$$

Given the vector $\left(\hat{\gamma}_{1}, \ldots, \hat{\gamma}_{m}\right)$ and given that the tax schedule $T(\cdot)$ takes the form $T(y)=\tau_{0}+\tau_{1} y$, I write $\hat{T}(y):=\hat{\tau}_{0}+\hat{\tau}_{1} y$, where

$$
\hat{\tau}_{0}:=\tau_{0} \sum_{i=1}^{m} p_{i} \hat{\gamma}_{i} \text { and }\left(1-\hat{\tau}_{1}\right):=\left(1-\tau_{1}\right) \sum_{i=1}^{m} p_{i} \hat{\gamma}_{i} .
$$

From the specifications of $T(\cdot)$ and $\hat{T}(\cdot)$, one obviously has

$$
y(t)-\hat{T}(y(t))=(y(t)-T(y(t))) \sum_{i=1}^{m} p_{i} \hat{\gamma}_{i}
$$

for all $t$. From (9) and (10), one sees that, for any $t$, the vector $\left(\hat{c}_{1}(t), \ldots, \hat{c}_{m}(t), y(t)\right)$ satisfies the budget constraint (2) when the tax schedule is $\hat{T}(\cdot)$ and the consumer prices are $p_{1}, \ldots, p_{m}$.

It remains to be shown that, for any $t$,

$$
u\left(c_{1}, \ldots, c_{m}, \frac{y}{t}\right) \leq u\left(\hat{c}_{1}(t), \ldots, \hat{c}_{m}(t), \frac{y}{t}\right),
$$

for any outcome $\left(c_{1}, \ldots, c_{m}, y\right)$ that satisfies the consumer's budget constraint when the tax schedule is $\hat{T}(\cdot)$ and the consumer prices are $p_{1}, \ldots, p_{m}$. By the definitions of $\left(\hat{\gamma}_{1}, \ldots, \hat{\gamma}_{m}\right)$ and of the tax schedule $\hat{T}(\cdot)$ and by homotheticity, any such outcome satisfies

$$
\begin{aligned}
\varphi\left(c_{1}, \ldots, c_{m}\right) & \leq \psi\left(\frac{y-\hat{T}(y)}{\sum_{i=1}^{m} p_{i} \hat{\gamma}_{i}}\right) \varphi\left(\hat{\gamma}_{1}, \ldots, \hat{\gamma}_{m}\right) \\
& =\psi(y-T(y)) \varphi\left(\gamma_{1}, \ldots, \gamma_{m}\right) \\
& =\varphi\left((y-T(y)) \gamma_{1}, \ldots,(y-T(y)) \gamma_{m}\right) .
\end{aligned}
$$


Because $\sum_{i=1}^{m} q_{i} \gamma_{i} \leq 1$, the vector $\left((y-T(y)) \gamma_{1}, \ldots,(y-T(y)) \gamma_{m}, y\right)$ satisfies the budget constraint (2). Because $T(\cdot)$ and $q_{1}, \ldots, q_{m}$ implement the allocation $\left(c_{1}(\cdot), \ldots, c_{m}(\cdot), y(\cdot)\right)$,

$$
u\left(\varphi\left((y-T(y)) \gamma_{1}, \ldots,(y-T(y)) \gamma_{m}, \frac{y}{t}\right) \leq u\left(c_{1}(t), \ldots, c_{m}(t), \frac{y}{t}\right) .\right.
$$

Upon combining (13) and (14) and using the fact that the allocations $\left(c_{1}(\cdot), \ldots\right.$, $\left.c_{m}(\cdot), y(\cdot)\right)$ and $\left(\hat{c}_{1}(\cdot), \ldots, \hat{c}_{m}(\cdot), y(\cdot)\right)$ generate the same utility, one obtains (12).

To conclude the proof of Theorem 1, I note that, because $u$ is strictly quasi-concave, the solution to the problem of maximizing $u$ under the constraint (2) is unique and depends continuously on the parameters of the tax schedule. A small reduction in $\hat{\tau}_{0}$ can therefore be used to redistribute some of the surplus that is available under the strictly feasible allocation $\left(\hat{c}_{1}(\cdot), \ldots, \hat{c}_{m}(\cdot), y(\cdot)\right)$. This makes everybody better off without violating feasibility.

\section{References}

[1] Atkinson, A.B., and J.E. Stiglitz (1976), The Design of Tax Structure: Direct versus Indirect Taxation, Journal of Public Economics 6, 55 - 75.

[2] Deaton, A. (1979), Optimally Uniform Taxes, Economics Letters 2, 357 $-361$.

[3] Guesnerie, R. (1995), A Contribution to the Pure Theory of Taxation, Econometric Society Monograph, Cambridge University Press, Cambridge, U.K.

[4] Hammond, P. (1979), Straightforward Individual Incentive Compatibility in Large Economies, Review of Economic Studies 46, 263 - 282.

[5] Kaplow, L. (2006), On the Undesirability of Commodity Taxation Even When Income Taxation Is Not Optimal, Journal of Public Economics $90,1235-1250$.

[6] Laroque, G. (2005), Indirect Taxation is Superfluous under Separability and Taste Homogeneity: A Simple Proof, Economics Letters 87, 141 144.

[7] Werning, I. (2007), Optimal Fiscal Policy With Redistribution, Quarterly Journal of Economics 122, 925 - 967. 